\title{
Recomposición de un rayo de luz blanca descompuesto por un prisma
}

\author{
Alejandro del Mazo Vivar (iD \\ I.ES. Francisco Salinas. Salamanca. España.mazovivar@gmail.com \\ Santiago Velasco Maíllo \\ Departamento de Física Aplicada. Universidad de Salamanca. Salamanca. España.santi@usal.es \\ Rafael García-Molina \\ Departamento de Fisica. Universidad de Murcia.Murcia.España.rgm@um.es
}

[Recibido: 25 marzo 2020. Revisado: 9 junio 2020. Aceptado: 15 junio 2020]

\begin{abstract}
Resumen: La recomposición de un rayo de luz blanca, descompuesto inicialmente por un prisma, mediante el uso de otro prisma idéntico e invertido respecto al anterior es imposible $y$, sin embargo, numerosas fuentes (bibliográficas y de internet) suelen presentar este supuesto experimento como una actividad de fácil ejecución e incuestionable resultado. En este artículo se muestra el uso de diferentes elementos ópticos para recomponer un rayo de luz blanca, previamente descompuesto por un prisma. Todos los materiales empleados son de fácil adquisición, y el montaje de los mismos no ofrece ninguna dificultad, lo que permite la realización de estos experimentos cuando se discute la refracción y reflexión de la luz.
\end{abstract}

Palabras clave: Dispersión cromática; Refracción de la luz; Óptica; Recomposición de luz blanca.

\section{Recomposition of a ray of white light previously decomposed by a prism}

The recomposition of a ray of white light, initially decomposed by a prism, by using another identical prism and inverted with respect to the former is impossible and, however, many (bibliographic and internet) sources usually present this supposed experiment as a demonstration of easy execution and unquestionable result. This article shows the use of different optical elements to recompose a ray of white light, previously decomposed by a prism. All the materials employed can be easily acquired and assembled, which allows the use of these experiments when discussing refraction and reflection of light.

Keywords: Chromatic dispersion; Light refraction; Optics; Recomposition of white light.

Para citar este artículo: Del Mazo Vivar A., Velasco Maíllo S. García-Molina R. (2020) Reconposición de un rayo de luz blanca descompuesto por un prisma. Revista Eureka sobre Enseñanza y Divulgación de las Ciencias 17(3), 3402. doi: 10.25267/Rev_Eureka_ensen_divulg_cienc.2020.v17.i3.3402

\section{Un error demasiado común}

En uno de los capítulos del libro de Frank Wilczek (2016) titulado El mundo como obra de arte, el autor aborda el asunto de los experimentos realizados por Newton sobre la descomposición cromática de la luz blanca con ayuda de prismas. Aquellos experimentos probaban que un prisma no "modifica" o "degrada" la luz blanca -como era la opinión más extendida en la época-, sino que la "analiza", poniendo de manifiesto su composición. De este modo, se evidenciaba que los colores que surgían del prisma eran en realidad los componentes de la luz blanca. Respecto a alguno de estos experimentos Wilczek (2016) escribe lo siguiente:

"Un experimento simple pero profundo, que Newton citó como el experimentum crucis (el "experimento crucial") para su propuesta, presenta el argumento visiblemente, como se ve en la Lámina J. Los colores espectrales, en los que la luz blanca es analizada por un prisma, pueden reensamblarse [sic] como luz blanca utilizando un segundo prisma... El fondo de la cuestión es que, con un segundo

\author{
Revista Eureka sobre Enseñanza y Divulgación de las Ciencias \\ Universidad de Cádiæ. APAC-Eureka. ISSN: 1697-011X \\ http://dx.doi.org/10.25267/Rev_Eureka_ensen_divulg_cienc.2020.v17.i3.3402 \\ http:/ / reuredcuca.es
}


prisma, podemos revertir la separación y regresar a la luz blanca, una luz indistinguible en sus propiedades de la luz solar con la que empezamos".

Una adaptación de la citada lámina J se muestra en la figura 1.
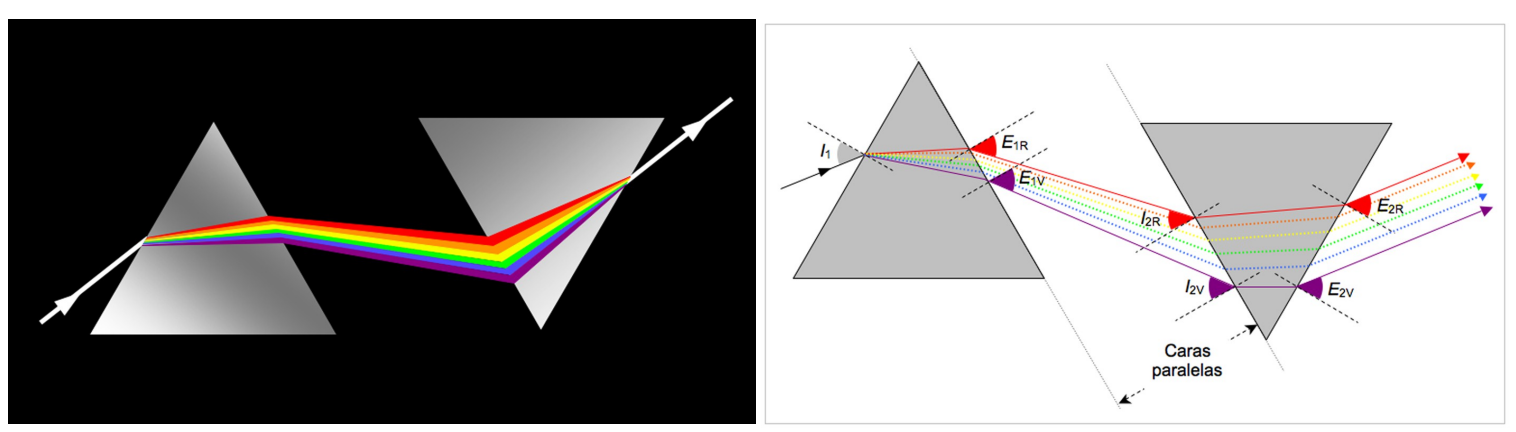

Figura 1. Adaptación de la lámina J de la obra de Figura 2. Formación del espectro visible con dos Wilczek (2016), donde se muestra el supuesto prismas ópticos, cuando el segundo está invertido $180^{\circ}$ comportamiento de los colores del espectro visible con respecto al primero. Debido a las refracciones que se dos prismas ópticos, cuando el segundo está invertido producen en los dos prismas, los diferentes colores $180^{\circ}$ respecto al primero. emergen finalmente separados. Los rayos que emergen (por la derecha) del segundo prisma son paralelos al rayo incidente (por la izquierda) de luz blanca, por lo que son también paralelos entre sí.

Wilzeck, Premio Nobel de Física en 2004, escribe el erróneo párrafo anterior, posiblemente influido por la reiterada -aunque incorrecta- afirmación de que los colores obtenidos por la descomposición de un rayo de luz blanca mediante un prisma se reagrupan por medio de otro prisma igual al primero, aunque girado $180^{\circ}$ respecto a él. Así aparece expuesto ya en manuales de Física de los siglos XIX y Xx (Gray 1851, Kaeppelin 1844, Ganot 1859, Mañas y Bonví 1935). Y esta idea sigue difundiéndose en nuestros días (Arora 2013, Tutor vista studio 2017, Vidal Fernández y Sánchez Gómez 2017, Rowlands 2017, College of Optometrists 2020). No estamos ante un hecho aislado, ya que en el terreno de la ciencia no son del todo infrecuentes las falsas creencias que se propagan sin verificación (Beaty 2020). El supuesto movimiento browniano de los granos de polen es otro buen ejemplo (Lavenda 1985, Hossenfelder 2019), desmentido en diversos foros (Wilkinson 2005, Del Mazo 2018). En el caso de los dos prismas, es posible que una impresión precipitada de simetría en la estructura del experimento sea la causante de ese error: "si los rayos de diferentes colores que abandonan el primer prisma fueran paralelos entre sí", al entrar en el segundo prisma experimentarían el fenómeno contrario, con la consiguiente reagrupación en luz blanca. Sin embargo, esto no es así, porque los rayos que salen del primer prisma no son paralelos (como se enfatiza mediante el entrecomillado), sino divergentes.

En los vidrios ópticos el índice de refracción disminuye al aumentar la longitud de onda (Tipler 1999). Para simplificar la discusión que sigue, se centrará la atención en el comportamiento de los colores que limitan el espectro visible que, de mayor a menor longitud de onda, son el rojo y el violeta. $\mathrm{Al}$ incidir un rayo de luz blanca con un ángulo $I_{1}$ sobre una cara de un prisma (figura 2), la ley de Snell explica que el rayo violeta se desvía más que el rojo al abandonar el prisma por una segunda cara. Los respectivos ángulos de emergencia son $E_{1 \mathrm{~V}} \mathrm{y}$ $E_{1 \mathrm{R}}$. Puesto que el segundo prisma es igual al primero y está girado $180^{\circ}$ respecto a él, el rayo violeta que llega a ese segundo prisma lo hace con ángulo de incidencia $I_{2 \mathrm{v}}$ igual a $E_{1 \mathrm{v}}$ y el rayo rojo con un ángulo $I_{2 \mathrm{R}}$ igual a $E_{1 \mathrm{R}}$. De nuevo, la ley de Snell establece los ángulos de emergencia en el segundo prisma, $E_{2 \mathrm{~V}}$ para la luz violeta y $E_{2 \mathrm{R}}$ para la roja, y cada uno de estos 
rayos ha de ser paralelo al rayo inicial de luz blanca. Si se amplía el razonamiento al resto de colores del espectro visible se concluye que todos ellos salen del segundo prisma paralelos, pero con trayectorias claramente separadas. Esto justifica que la luz blanca descompuesta por un prisma no se pueda recomponer mediante un segundo prisma idéntico girado $180^{\circ}$ respecto al primero (Jargodzki y Potter 2001, García Molina 2002). En García-Molina, Del Mazo y Velasco (2018) y Del Mazo, Velasco y García-Molina (2018) puede encontrarse una detallada discusión de la falacia anterior, así como un montaje que claramente muestra la imposibilidad de la recomposición de la luz blanca en las condiciones antes expresadas. En la figura 3 se aprecia claramente que la luz que emerge del segundo prisma consiste en los haces paralelos de los colores constituyentes de la luz que incide sobre el primer prisma. En todas las fotografías de los autores que se presentan a continuación (excepto la de la figura 13), el rayo de luz blanca incide horizontalmente por la parte superior izquierda.

En ocasiones, se sostiene que la recomposición de la luz blanca con dos prismas idénticos sí es posible cuando estos se encuentran muy próximos. Es fácil entender que no existe una distancia de separación mínima entre prismas a partir de la cual ya hay recomposición de colores. Lo que sucede es que los rayos coloreados que emergen del primer prisma llegan al segundo con muy poca separación entre ellos, por lo que siguen estando muy próximos a la salida de este último prisma. Si se hace una observación cuidadosa del supuesto rayo blanco emergente, como la que aparece en la figura 4, se percibe una gradación cromática en los bordes del mismo (Avison 1989, Alonso y Finn 1992).
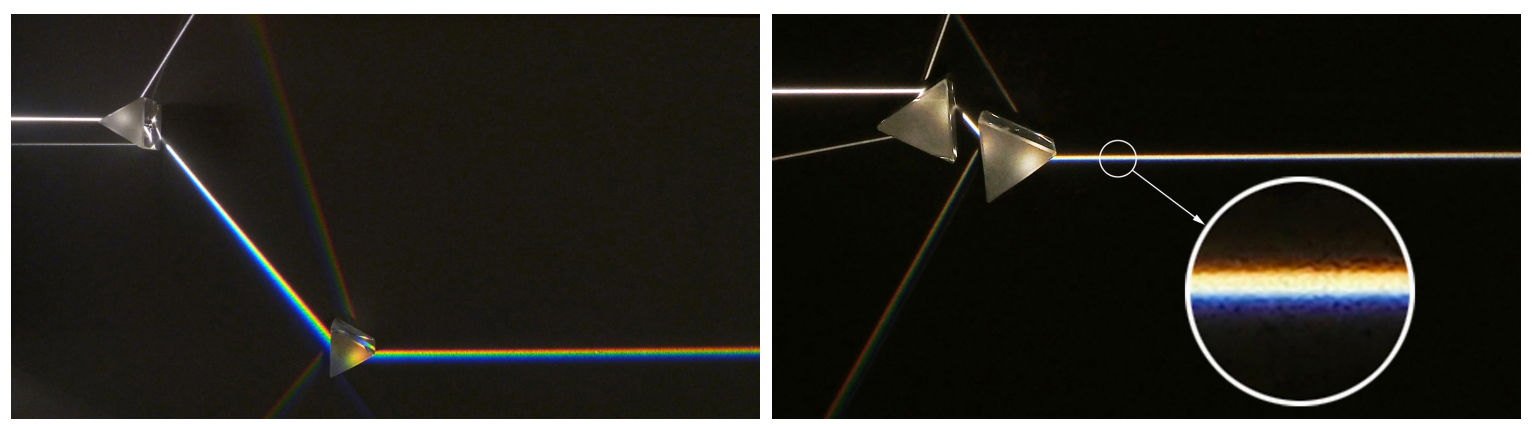

Figura 3. Un rayo de luz blanca que incide en un Figura 4. En un montaje con dos prismas muy primer prisma se refracta, es descompuesto en sus próximos parece producirse recomposición de la luz colores constituyentes y llega a un segundo prisma blanca. Una observación cuidadosa muestra que el rayo invertido respecto al primero. Los diferentes colores que emerge del segundo prisma presenta en sus lados que emergen del segundo prisma son paralelos al rayo los colores de ambos extremos del espectro visible. inicial. La fotografía muestra también las reflexiones y refracciones secundarias que tienen lugar en las distintas caras de los prismas.

Algunos autores, como Greenslade (1984), han sustentado esta supuesta recomposición en textos de Física del siglo XIX, como el de Guillemin (1868). Este, a pesar de la errónea representación que hace de los rayos de luz a la salida del primer prisma (figura 5), sí reconoce que el rayo que emerge del segundo prisma tiene su borde superior de color rojo y el inferior de color violeta, tal como corresponde a la luz blanca descompuesta procedente del primer prisma, la cual incide en el segundo prisma. Otros autores presentan un dispositivo experimental similar (figura 6) para justificar la recomposición de la luz blanca (Lozano 1898), sin ni siquiera prestar atención al detalle de los bordes coloreados citado anteriormente.

Sin embargo, en otras obras de ese mismo siglo, se hace ver con bastante claridad la imposibilidad de la recomposición usando esa configuración de prismas. Así, Feliu (1883 p. 329) afirma: 
"Si en vez de un manojo de rayos fuera posible emplear un rayo lineal, el espectro se produciría aun después de atravesar los prismas, porque los colores no se superpondrían, quedando el rojo a cierta distancia del violado, por más que fuesen paralelos, a consecuencia de la desviación lateral. Aún en el ensayo precedente se nota que el borde superior de la imagen blanca es rojo y el inferior violado."
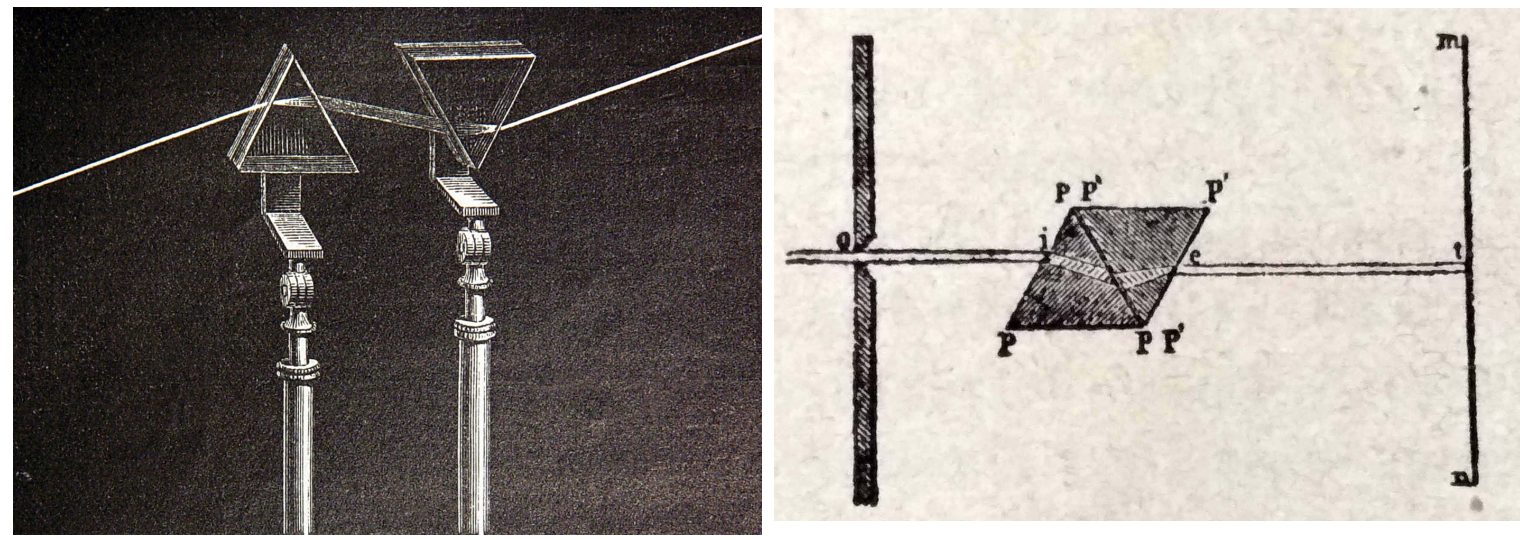

Figura 5. Supuesto comportamiento de la luz blanca Figura 6. Disposición de dos prismas idénticos, uno en un montaje con dos prismas, según Guillemin invertido respecto del otro, para "reunir los colores (1868). Los rayos que se propagan entre esos prismas dispersados por el prisma P" en un rayo de luz blanca aparecen paralelos, cuando en realidad son divergentes. et paralelo a oi (Lozano 1898).

Finalmente, el rayo que emerge del segundo prisma tiene (erróneamente) igual anchura que el rayo blanco incidente.

\section{¿Es posible recomponer los colores?}

Como se ha dicho anteriormente, el mito de la recomposición de los colores del espectro visible en luz blanca utilizando dos prismas iguales, invertidos entre sí $180^{\circ}$, ha alcanzado cierta difusión y se ha atribuido frecuentemente a Newton, quien no menciona tal recomposición al realizar el experimento de los dos prismas citado previamente (Newton 1671), ni tampoco aparece referida en un comentario coetáneo de este experimento (Moray 1672). No obstante, en lo que sigue se mostrará que sí existen formas de conseguir la recomposición de los colores, algunas de las cuales ya fueron descritas por Newton (1730).

El equipo experimental elegido para realizar los montajes consta de un foco con una lámpara incandescente de $20 \mathrm{~W}$, con un filamento recto perpendicular a la superficie de trabajo. Su luz incide de forma rasante sobre una superficie blanca en la que se apoyan los elementos ópticos empleados en los diferentes dispositivos experimentales. Al interponer en el camino de la luz una cartulina negra con una rendija vertical de $0,5 \mathrm{~mm}$ de ancho, aproximadamente, se consigue un estrecho haz de luz con pequeña divergencia. El resto del material está formado por varios prismas equiláteros de vidrio crown de $2,3 \mathrm{~cm}$ de lado, una sección de lente convergente biconvexa de $5,0 \mathrm{~cm}$ de longitud y $2,0 \mathrm{~cm}$ de espesor, cuya distancia focal es de $3,2 \mathrm{~cm}$ y una sección de espejo cóncavo de $7,0 \mathrm{~cm}$ de longitud, con una distancia focal de 2,6 $\mathrm{cm}$. Con estos componentes se realizarán los montajes que se describen a continuación, que han de efectuarse con baja luminosidad ambiental.

Montaje $\mathbf{1}^{\mathbf{}}$. Se parte de la configuración mostrada en la figura 3 , en la que los rayos de los diferentes colores del espectro visible emergen paralelos del segundo prisma. Cuando estos rayos inciden sobre una lente convergente, se refractan y convergen en su foco (Sanjurjo 1883, Feliu 1883 p. 323, Marcoláin 1912); en sentido estricto hay que admitir que tanto las 
aberraciones ópticas de los componentes utilizados como la leve divergencia que presenta el haz de luz blanca original impiden que todos los rayos converjan rigurosamente en un mismo punto. Estas circunstancias han de tenerse en cuenta para el resto de los montajes propuestos. De este modo se consigue recomponer la luz blanca en un solo punto del espacio, que es el indicado en la figura 7 por $F^{\prime}$ (el foco posterior de la lente).

Montaje $2^{\mathbf{o}}$. Esta disposición es parecida a la del montaje anterior. La diferencia reside en que el haz paralelo de colores que emerge del segundo prisma incide sobre un espejo cóncavo (Marcoláin 1912). De este modo, los rayos se reflejan y convergen en el foco del espejo, donde se obtiene un punto de luz blanca (figura 8). Girando el espejo, la convergencia se consigue en un punto separado de su eje óptico, lo que facilita la visibilidad.

Montaje $3^{\mathbf{o}}$. Se parte nuevamente del montaje de la figura 3. Si los rayos paralelos que emergen del segundo prisma inciden sobre un tercer prisma que presenta simetría vertical respecto al segundo prisma (según el plano de la figura), los rayos convergen (figura 9). La posición del punto en el que se obtiene la luz blanca está alejada y es algo confusa, ya que los rayos que emergen del tercer prisma muestran menor convergencia que en los dos montajes citados anteriormente (Newton 1730 Book I Part II Prop. V Theor. IV Exper. 10).

Montaje $4^{\circ}$. Se hace llegar un estrecho haz de luz blanca sobre un prisma. Si el haz divergente de colores que abandona el prisma incide sobre una lente convergente, se consigue que converja, en forma de luz blanca, en un punto, $P$, situado a una distancia $d$ del vértice de la segunda superficie de la lente, que es mayor que la distancia focal posterior, s' (figura 10) (Newton 1730 Book I Part II Prop. I Theor. I Exper. 2).
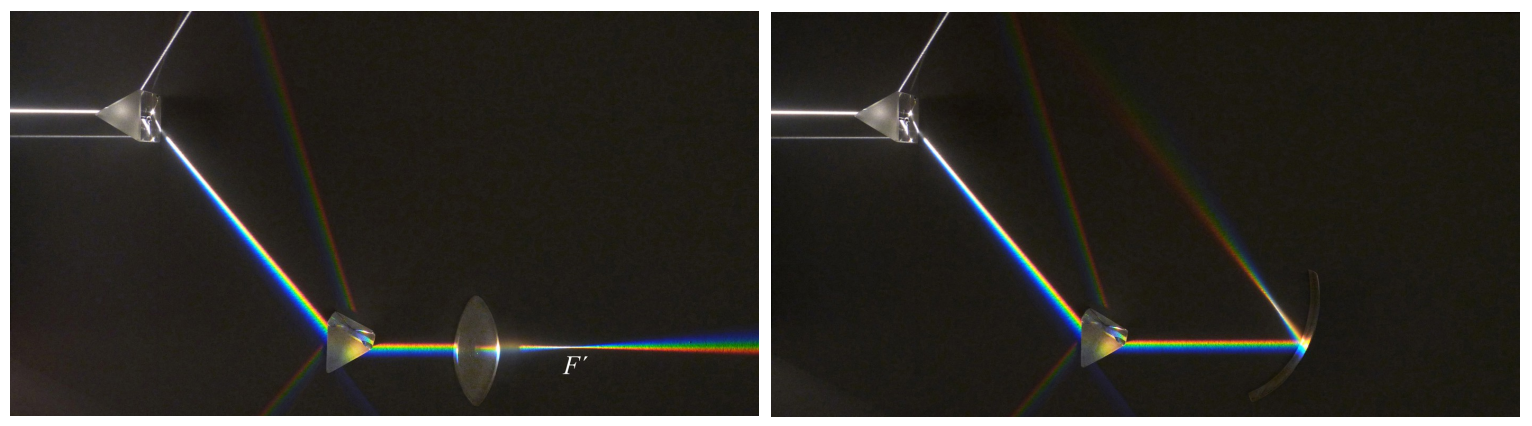

Figura 7. El haz de rayos coloreados que emerge del Figura 8. La configuración de componentes es muy montaje con dos prismas llega a una lente biconvexa. parecida a la del montaje $1^{\circ}$. La diferencia está en que Esos rayos se refractan pasando por el foco, $F$ ', donde se ha sustituido la lente biconvexa por un espejo se obtiene luz blanca. cóncavo. Al girar el espejo se hace más visible el punto en el que se produce la convergencia y la consiguiente luz blanca.

Montaje $\mathbf{5}^{\mathbf{}}$. Se toma nuevamente, como punto de partida el montaje de la figura 3. Los prismas se sitúan con una separación próxima a $4 \mathrm{~cm}$ entre sus centros y el segundo de ellos se gira un ángulo cercano a $30^{\circ}$ en sentido antihorario. En estas condiciones, los rayos de los diferentes colores que abandonan el segundo prisma muestran una convergencia débil y crean, en una posición alejada de este prisma, la sensación de luz blanca (figura 11). Esta percepción tiene lugar debido a que los rayos de los diferentes colores se encuentran bastante próximos, a pesar de no converger estrictamente en el mismo punto. 

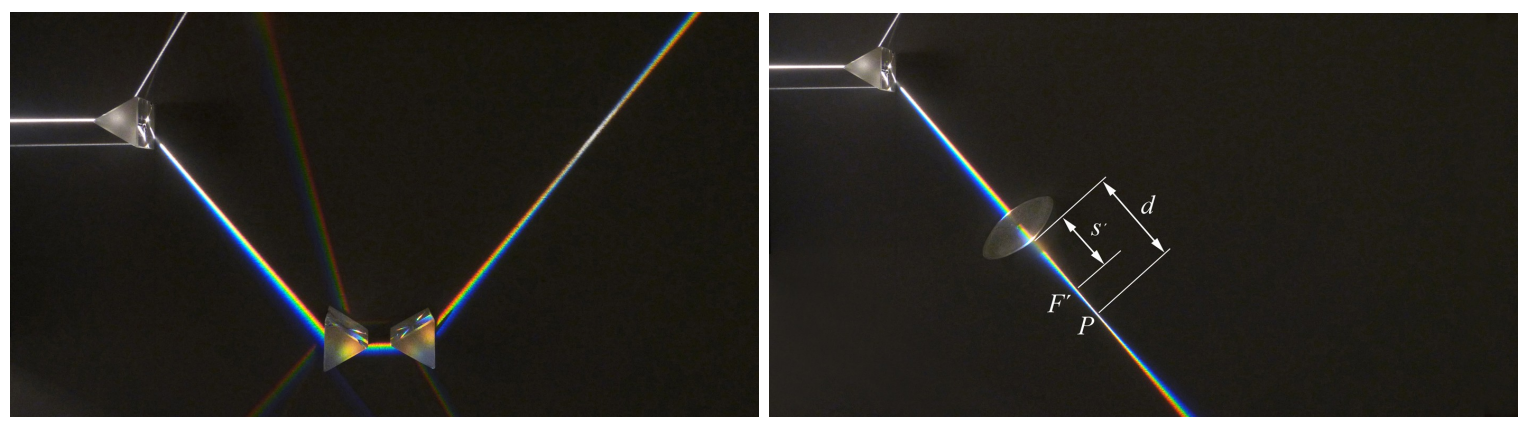

Figura 9. A diferencia de los montajes $1^{\circ}$ y $2^{\circ}$, el haz Figura 10. Un rayo de luz blanca incide sobre un paralelo de rayos coloreados que emerge del montaje prisma y el espectro de colores que emerge se hace con dos prismas llega a un tercer prisma con simetría llegar sobre una lente biconvexa. Los colores vertical (según el plano de la figura) respecto al convergen en un punto, $P$, que se encuentra a una segundo prisma. El lugar preciso donde se produce la distancia $d$ mayor que la distancia focal posterior, $s^{\prime}$. En convergencia de los rayos es algo confuso. ese punto se consigue luz blanca.

En todos los montajes anteriores se ha conseguido que los colores constituyentes de la luz blanca se recompongan solo en un punto del espacio. Se verá a continuación que también es posible obtener nuevamente un haz de luz blanca mediante disposiciones simétricas de los componentes ópticos.

Montaje $\mathbf{6}^{\mathbf{0}}$. Se parte nuevamente de la configuración de la figura 3. Cuando los rayos coloreados que emergen del segundo prisma inciden sobre una pareja de prismas idéntica a la primera pareja, de tal manera que el conjunto muestre simetría vertical (según la figura 12), se produce una distribución de rayos de luz que también es simétrica, de modo que del último prisma emerge un rayo de luz blanca (Jargodzki y Potter 2001 p. 169 \#58).

Montaje $7^{\mathbf{0}}$. Un estrecho haz de luz blanca llega a un prisma y se refracta. El haz divergente de colores incide en una lente biconvexa simétrica y los rayos que emergen de la lente llegan a un segundo prisma idéntico al primero, que está dispuesto simétricamente respecto a él (en relación al eje de simetría de la lente). En este caso, del segundo prisma emerge un rayo de luz blanca, tal como se aprecia en la figura 13 (Newton 1730 Book I Part II Prop. XI Prob. VI). Como curiosidad, este es el montaje que se ha popularizado en un cómic que alude a la portada de un famoso disco de Pink Floyd (Munroe 2011).
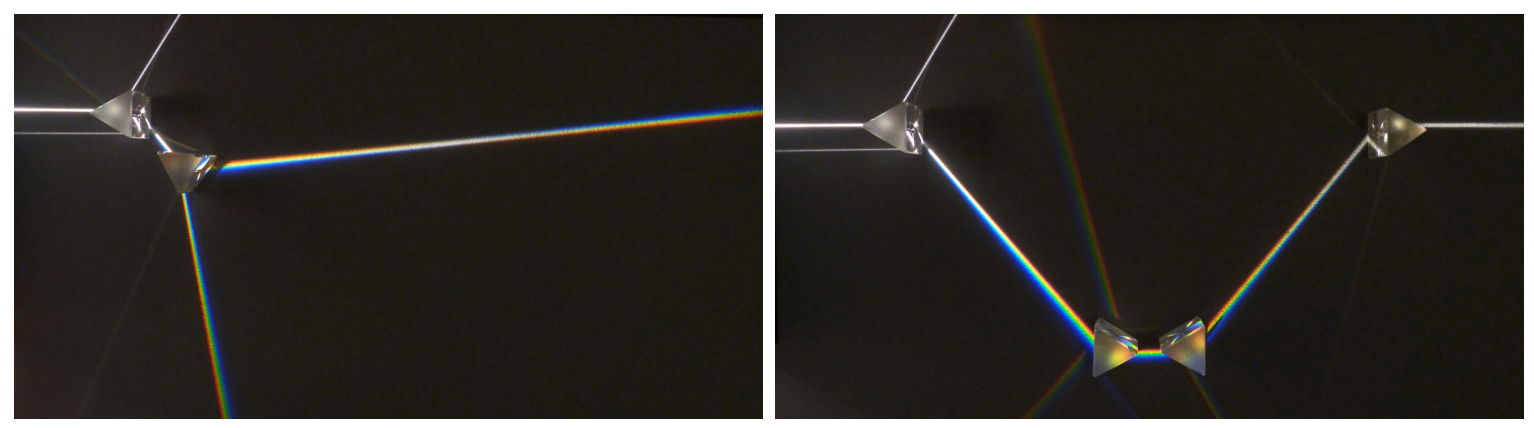

Figura 11. Inicialmente se parte de un montaje Figura 12. Si se parte de la pareja de prismas de la análogo al de la figura 3. Si se gira el segundo prisma figura 3 y se añade otra nueva pareja de prismas con en sentido antihorario, los colores que emergen de este simetría vertical respecto a la primera, se consigue prisma convergen hacia una posición alejada del también que los rayos de luz muestren simetría. Por mismo. De nuevo, el lugar preciso en el que se tanto, del cuarto prisma emerge un rayo de luz blanca. produce la convergencia es algo confuso. 


\section{Resumen y conclusiones}

Se ha mostrado que los colores obtenidos por la descomposición de un rayo de luz blanca no pueden agruparse para crear nuevamente luz blanca si solo se utiliza un segundo prisma, invertido respecto al primero e idéntico a él.

No obstante, se han expuesto otros procedimientos con objeto de obtener esa recomposición de luz blanca, en unos casos convergiendo en un punto del espacio y, en otros, formando nuevamente un haz de luz blanca. Los diversos montajes se han conseguido, tanto mediante la combinación de prismas y lentes o espejos curvos, como

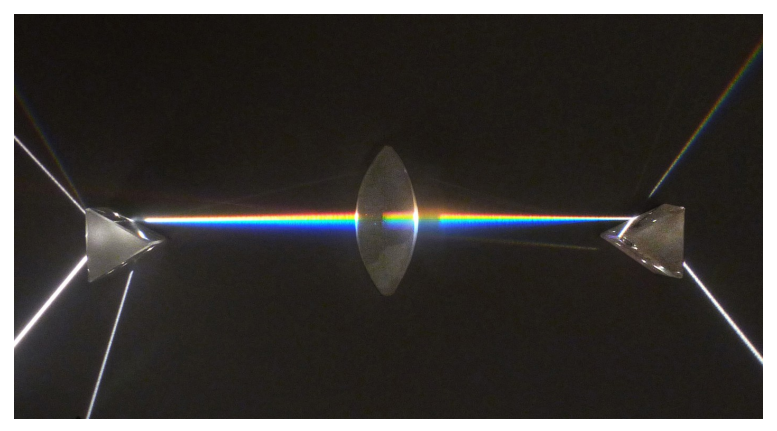

Figura 13. Una nueva configuración simétrica conduce a la obtención de un rayo de luz blanca que sale del prisma de la derecha en sentido descendente después de incidir un rayo de luz blanca en sentido ascendente sobre el prisma de la izquierda. Las distancias entre las caras de los dos prismas orientadas hacia la lente biconvexa y las empleando dos prismas iguales (aunque correspondientes caras de esa lente son iguales y muy girados un ángulo diferente de $180^{\circ}$ ) o con próximas al doble de su distancia focal.

tres o cuatro prismas iguales.

Las posibilidades para alcanzar este propósito no se limitan a los ejemplos expuestos. Existen otras formas para agrupar los colores que emergen de un prisma tras descomponer la luz blanca. Por ejemplo, el uso de prismas con diferente índice de refracción, o elementos ópticos más sofisticados, no disponibles usualmente en un laboratorio de física general (Pregger 1982, McDonald 2018), abre otras vías para recomponer los colores del espectro visible que no han sido tratadas aquí.

Dado que en los experimentos de óptica hay un delicado balance entre la experiencia sensorial y el trazado de rayos ópticos, no se han considerado otras alternativas, basadas en la percepción de luz blanca como resultado de la respuesta fisiológica del ojo humano a la recepción de diferentes colores, lo cual puede dar lugar a apreciaciones confusas.

Estas actividades son fáciles de implementar durante la discusión de la dispersión cromática de la luz blanca, estimulando la creatividad de estudiantes y profesorado para conseguir recomponer la luz blanca mediante el empleo de diferentes elementos ópticos.

\section{Referencias}

Alonso M., Finn E. J. (1992) Physics, Wokingham: Addison-Wesley. Fig. 33.29a.

Arora A. (2013) Recombination of White Light (JAL 15A) Video lectures by Ashish Arora.

Avison J. (1989) The World of Physics, $2^{\mathrm{a}}$ ed. Cheltenham: Thomas Nelson and Sons Ltd. p. 31 Fig. 2.11.

Beaty B. (2020) Science Myths, en K-6 Textbooks and Popular culture. Science Hobbyist. Recuperado de http://amasci.com/miscon/miscon.html

College of Optometrists (2020) Newton and the colour of light.

Del Mazo A., Velasco S., García-Molina R. (2018) El mito de la recomposición de la luz blanca con dos prismas.

Del Mazo A. (2018) El mito del movimiento browniano del polen. 
Feliu y Pérez B. (1883) Física experimental y aplicada, y nociones de química, $5^{\mathrm{a}}$ ed. Barcelona: Imprenta de Jaime Jepús. p. 323.

Feliu y Pérez B. (1896)Tratado elemental de Física experimental y aplicada, $8^{a}$ ed. Zaragoza: Tipografía de Comas hermanos. p. 329.

Ganot A. (1859) Traité élémentaire de physique, expérimentale et appliquée, 13a ed. París: Ganot. p. 487.

García Molina R. (2002) ¿Recomposición de la luz blanca? Cuestión 23 de Simple+mente física.

García-Molina R., Del Mazo A., Velasco S. (2018) A Simple Experimental Setup to Clearly Show that Light Does Not Recombine After Passing Through Two Prisms, The Physics Teacher 56 (1), 14-16.

Gray A. (1851) Natural Philosophy. New York: Harper \& Brothers. p. 359.

Greenslade T. (1984) Spectrum recombination, The Physics Teacher 22 (2), 105-108.

Guillemin A. (1868) Les phénomènes de la Physique. París: Librería de L. Hachette et Cie. p. 353.

Hossenfelder S. (2019) Perdidos en la matemáticas. Barcelona: Ariel. p.64

Jargodzki C., Potter F. (2001) Mad about Physics. Braintwisters, Paradoxes, and Curiosities. Nueva York: Wiley.

Kaeppelin R. (1844) Cours élémentaire des sciences physiques. Cours de Physique, $3^{\mathrm{a}}$ ed. París: Z. Kaeppelin. p. 412.

Lavenda B. (1985) El movimiento browniano. Investigación y Ciencia 103, 36-45.

Lozano y Ponce de León E. (1898) Elementos de Física, 6a ed. Barcelona: Imprenta de Jaime Jepús y Roviralta. p. 473 Fig. 335.

Mañas y Bonví J. (1935) Óptica aplicada, $2^{\text {a }}$ ed. Barcelona: Altés. p. 324.

Marcoláin San Juan P. (1912) Elementos de Física moderna y nociones de meteorología. Zaragoza: Tipografía La Editorial. p. 514.

McDonald K. (2018) Recombining rainbows, The Physics Teacher 56 (4), 196-197.

Moray R. (1672) Some Experiments propos'd in relation to Mr. Newton's Theory of light ... together with the Observations made thereupon by the Author of that Theory. Philosophical Transactions of the Royal Society 83 (20 May 1672) 4059-4062.

Munroe R. (2011) Comic 964 Dorm Poster.

Newton I. (1671) A Letter of Mr. Isaac Newton ... containing his New Theory about Light and Colors. Philosophical Transactions of the Royal Society 80 (19 Feb. 1671/2) 3075-3087.

Newton I. (1730) Opticks, $4^{a}$ ed. Londres: William Innys [reeditado por Dover en 1952].

Pregger F. T. (1982) Recombination of spectral colors. The Physics Teacher 20 (9), 403.

Rowlands P. (2017) Newton and Modern Physics. London: World Scientific. p. 111

Sanjurjo R. (1883) Principios fundamentales de Física pura, Tomo I. Madrid: Imprenta y Litografía de La Guirnalda. p. 418 Fig. 439.

Tipler P. A. (1999) Física para la ciencia y la tecnología, Vol. 2, 4ª ed. Barcelona: Reverté. p.1094.

Tutor vista studio (2017) Dispersion of White Light by Glass Prism [Vídeo] 
Vidal Fernández, M. C., Sánchez Gómez D. (2017) Física. $2^{\circ}$ Bachillerato. Madrid: Santillana. p. 206.

Wilczek F. (2016) El mundo como obra de arte. En busca del diseño profundo de la naturaleza. Barcelona: Crítica. p. 100.

Wilkinson D. (2005) Brown knew particles were smaller than pollen. Nature 434, 137. 